See discussions, stats, and author profiles for this publication at: https://www.researchgate.net/publication/289619841

\title{
Structural stability and normal forms of piecewise smooth vector fields on $\mathrm{R}-3$
}

Article in Publicationes mathematicae · January 2015

DOI: 10.5486/PMD.2015.5948

2 authors:

20. T. de Carvalho

São Paulo State University

47 PUBLICATIONS 145 CITATIONS

SEE PROFILE
READS

107

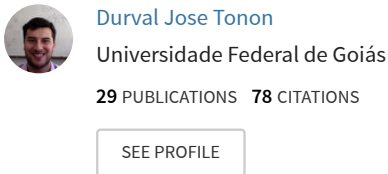

Some of the authors of this publication are also working on these related projects:

Modelagem Matemática e Computacional do Câncer e suas Terapias View project

Postdoctoral position at Pos graduate program in Mathematics of the Federal University of Goias View project 


\title{
STRUCTURAL STABILITY AND NORMAL FORMS OF PIECEWISE SMOOTH VECTOR FIELDS ON $\mathbb{R}^{3}$
}

\author{
TIAGO DE CARVALHO ${ }^{1}$ AND DURVAL J. TONON ${ }^{2}$
}

\begin{abstract}
The main goal of this paper is to exhibit normal forms of generic locally structurally stable piecewise smooth vector fields on $\mathbb{R}^{3}$. Besides, we construct the homeomorphism that gives the topological equivalence between an element of a locally structurally stable subset of piecewise smooth vector fields and its respective $C^{0}$-normal form.
\end{abstract}

\section{INTRODUCTION}

The overall goal of this work is to study some qualitative and geometric aspects of piecewise smooth vector fields (PSVF's for short) theory. PSVF's is a subject that has been developed at a very fast pace in recent years and it has become certainly one of the most common frontiers between Mathematics and Physics/Engineering. We consider vector fields expressible in the form $\dot{x}=Z(x)$ where $x \in \mathbb{R}^{3}$ is a state vector and $Z$ is a smooth piecewise mapping. The discontinuities are concentrated on a codimensionone submanifold $\Sigma$ of $\mathbb{R}^{3}$. Orbit-solutions on the switching manifold $\Sigma$ are defined according to Filippov's convention (see [8]). We point out that trajectories can be constrained to the switching manifold, and this behavior is termed sliding.

Recently, a lot of papers, see $[1,2,3,4,5,9,12]$ for example, exhibit local $\mathrm{C}^{0}$-normal forms of PSVF's and study some properties of them. We observe that the term normal form is referring to a representative system taken as simple as possible in each class. The use of such normal forms only makes sense if they represent a whole class of PSVF's presenting certain characteristics inherent on its configuration. In order to prove that the normal forms used represent the chosen class of PSVF's it is necessary to build homeomorphisms that preserve the dynamical properties of the vector fields involved. However, even if the definition of bifurcation is based on breaking structural stability, as long as we know, just in $[1,2,3,5,9,13]$ the authors effectively show how to construct the homeomorphisms which lead to equivalences between planar PSVF's presenting some intrinsic properties and the chosen $\mathrm{C}^{0}$-normal forms.

2010 Mathematics Subject Classification. 34A36, 34C23, 34D30, 34F10, 37G05.

Key words and phrases. normal forms, structural stability, singularity, piecewise smooth vector fields, bifurcation. 
Another important reference about normal forms and construction of homeomorphisms involving PSVF's on $\mathbb{R}^{3}$ is the pioneering book [8]. In fact, in the present paper we revisited and improved the list of normal forms establish on it. Moreover, some new ideas are considered and we proved that some of the cases covered here are not predicted by the normal forms in [8] (see Subsections 4.4.1, 4.4.2 and 4.4.3). Furthermore, here we explicitly construct the equivalence between the normal form and a PSVF that present the same topological structure. We obtain 26 topological distinct normal forms (see Theorems B and C), and present a set of PSVF's that is structural stable (see Theorem A). We believe that the construction of such homeomorphisms is a missing point in the literature about PSVF's, particularly in $\mathbb{R}^{3}$, and we hope to fill this gap for a class of codimension zero PSVF's on $\mathbb{R}^{3}$.

The most relevant difference between our approach and that one showed in [8] is that in [8] the author did not consider the tangencies of the trajectories of the sliding vector fields with the boundaries of the sliding region (see Lemmas 2, 3 and 4). Besides, in an oppositive way that showed in [8], we avoid the case where the PSVF has a double invisible tangency with $\Sigma$ because, by some recent results (see $[6,7,10]$ ), we get that this case is not structurally stable. In fact, the dynamics of $Z$ in this case can be chaotic [7], can presents non-trivial minimal sets (a cone of periodic orbits) [6], reversibility [10] or even asymptotic stability [11].

The paper is organized as it follows: In Section 2 we give some definitions, basic concepts and establish the notation. In Section 3 the main results are stated. In Section 4 are discussed some auxiliary results that prove the main results.

\section{BASIC THEORY}

2.1. Piecewise smooth vector fields. Let $\Sigma=h^{-1}(0)$, where $h$ is (a germ of) a smooth function $h:\left(\mathbb{R}^{3}, 0\right) \rightarrow(\mathbb{R}, 0)$ having $0 \in \mathbb{R}$ as a regular value. Designate by $\mathfrak{X}^{r}$ the space of all germs of $C^{r}$-vector fields on $\mathbb{R}^{3}$ endowed with the $C^{r}$-topology with $r>1$ large enough for our purposes. Call $\Omega^{r}=\mathfrak{X}^{r} \times \mathfrak{X}^{r}$ the space of all germs of vector fields $Z \in\left(\mathbb{R}^{3}, 0\right)$ such that

$$
Z(q)=\left\{\begin{array}{l}
X(q), \text { if } h(q) \geq 0 \\
Y(q), \text { if } h(q) \leq 0
\end{array}\right.
$$

Let $\Sigma_{+}=h^{-1}((0, \infty))$ and $\Sigma_{-}=h^{-1}((-\infty, 0))$. We will use the notation $Z=(X, Y)$ and consider $\Omega^{r}=\mathfrak{X}^{r} \times \mathfrak{X}^{r}$ endowed with the product topology. For each $X \in \mathfrak{X}^{r}$ we define

$$
X h(p)=\langle X(p), \nabla h(p)\rangle \quad \text { and } \quad X^{i} h(p)=\left\langle X(p), \nabla X^{i-1} h(p)\right\rangle, i \geq 2
$$

where $\langle.,$.$\rangle is the canonical inner product in \mathbb{R}^{3}$. We distinguish the following regions on $\Sigma$, according to the Filippov's convention, see [8]: 
- Sewing Region: $\Sigma^{c}=\{p \in \Sigma ;(X h)(p)(Y h)(p)>0\}$. Moreover, we denote $\Sigma^{c+}=\{p \in \Sigma ;(X h)(p)>0,(Y h)(p)>0\}$ and $\Sigma^{c-}=$ $\{p \in \Sigma ;(X h)(p)<0,(Y h)(p)<0\}$.

- Sliding Region: $\Sigma^{s}=\{p \in \Sigma ;(X h)(p)<0,(Y h)(p)>0\}$.

- Escaping Region: $\Sigma^{e}=\{p \in \Sigma ;(X h)(p)>0,(Y h)(p)<0\}$.

Let $\mathcal{O}=\Sigma^{s} \cup \Sigma^{e} \cup \Sigma^{c}$. It is well know that $\mathcal{O}$ is an open and dense subset of $\Sigma$. In particular, observe that given $p \in \mathcal{O}$ then $X(p) \neq 0$ and $Y(p) \neq 0$. The sliding vector field associated to $Z \in \Omega^{r}$ is the vector field $\bar{Z}^{\Sigma}$ tangent to $\Sigma^{s}$ and defined explicitly, at $q \in \Sigma^{s}$, by

$$
\bar{Z}^{\Sigma}=\frac{1}{\langle(Y-X), \nabla h\rangle}[\langle Y, \nabla h\rangle X-\langle X, \nabla h\rangle Y]=\frac{Y h X-X h Y}{Y h-X h} .
$$

The future orbit of $\bar{Z}^{\Sigma}$ coincides with the future orbit of the vector field

$$
Z^{\Sigma}=Y h X-X h Y,
$$

that will be called normalized sliding vector field. Note that $Z^{\Sigma}$ can be $C^{r}$-extended to the boundary of $\Sigma^{s}$.

Definition 1. The flow of $Z \in \Omega^{r}$ is obtained by the concatenation of flows of $X, Y$ and $Z^{\Sigma}$.

The concept of structural stability on $\Omega^{r}$ is based on the following definition:

Definition 2. Two vector fields $Z, \widetilde{Z} \in \Omega^{r}$, defined in neighborhood $U$ of $0 \in \mathbb{R}^{3}$, are $\Sigma$-equivalent if there exists an orientation preserving homeomorphism $\xi: U \rightarrow U$ that sends orbits of $Z$ to orbits of $\widetilde{Z}$, preserving $\Sigma$.

2.2. Singularities. The study of tangencies of trajectories of $X, Y$ and $Z^{\Sigma}$ with the boundary of $\Sigma$ is central to understand the dynamic nearby the switching manifold. In this way we say that $p \in \Sigma$ is a:

- regular point of $X \in \mathfrak{X}^{r}$ if $X h(p) \neq 0$.

- fold point of $X \in \mathfrak{X}^{r}$ if $X h(p)=0$ and $X^{2} h(p) \neq 0$.

- cusp point of $X \in \mathfrak{X}^{r}$ if $X h(p)=X^{2} h(p)=0, X^{3} h(p) \neq 0$ and $\left\{d h(p), d(X h)(p), d\left(X^{2} h\right)(p)\right\}$ is a linearly independent set.

- two-fold singularity of $Z \in \Omega^{r}$ if $X h(p)=Y h(p)=0, X^{2} h(p) \neq 0$ and $Y^{2} h(p) \neq 0$ (i.e., $p$ is a fold point of both $X$ and $Y$ ).

We say that $p \in \Sigma$ is a $\Sigma$-regular point of $Z \in \Omega^{r}$ if

(a) either $p \in \Sigma^{c} \cup \Sigma^{e}$ or

(b) $p \in \Sigma^{s}$ and $Z^{\Sigma}(p) \neq 0$ (i.e., $p \in \Sigma^{s}$ and $X(p)$ and $Y(p)$ are not parallel).

The points of $\Sigma$ which are not $\Sigma$-regular are called $\Sigma$-singular. We distinguish two subsets in the set of $\Sigma$-singular points: $\Sigma^{t}$ and $\Sigma^{p}$. Any $p \in \Sigma^{p}$ is called a pseudo equilibrium of $Z$ and it is characterized by $Z^{\Sigma}(p)=0$. Any $p \in \Sigma^{t}$ is called a tangential singularity and is characterized by $Z^{\Sigma}(p) \neq 0$ 
and $(X h(p))(Y h(p))=0$ (in this case the trajectories of $X$ or $Y$ are tangent to $\Sigma)$. Since $S_{X}=\{p \in \Sigma ; X h(p)=0\}$ is a codimension one set in $\Sigma$, we call it the fold curve of $X$ (the same for $Y$ ). In this way, given $Z=(X, Y)$ we get $\Sigma^{t}=S_{X} \cup S_{Y}$.

In what follows, we will fix a system of coordinates such that $h(x, y, z)=z$ and the fold curves are given, implicitly, by $S_{X}=\xi_{X}^{-1}(0)$ and $S_{Y}=\xi_{Y}^{-1}(0)$ where $\xi_{X}(x, y)=x$ and $\xi_{Y}(x, y)=y$. So, we define the following subsets of $\Omega^{r}$ :

- $\Omega_{1}=\left\{Z \in \Omega^{r} ; 0\right.$ is a regular point of both $X$ and $Y$ and either it is a $\Sigma$-regular point or a hyperbolic pseudo equilibrium $\}$;

- $\Omega_{2}=\left\{Z \in \Omega^{r} ; 0\right.$ is a fold point of $X$ and regular for $\left.Y\right\}$;

- $\Omega_{3}=\left\{Z \in \Omega^{r} ; 0\right.$ is a cusp point of $X$ and regular for $\left.Y\right\}$;

- $\Omega_{4}=\left\{Z \in \Omega^{r} ; 0\right.$ is a two-fold singularity, the eigenvectors of $D Z^{\Sigma}(0)$ are transverse to $S_{X}$ and $S_{Y}$ at 0 and 0 is a hyperbolic critical point of $\left.Z^{\Sigma}\right\}$.

Let

$$
\Omega_{0}=\Omega_{1} \cup \Omega_{2} \cup \Omega_{3} \cup \Omega_{4} .
$$

By $[14,17]$ and observing that the intrinsic conditions involving the sets $\Omega_{i}$, $i=1,2,3,4$ are generic, we conclude that $\Omega_{0}$ is an open and dense subset of $\Omega^{r}$. Note that the dynamic of $Z \in \Omega_{4}$ depends on the sign of $X^{2} h(0)$ and $Y^{2} h(0)$, see Figure 1. Along this paper we consider the following partition on $\Omega_{4}$ :

(a) Parabolic case: either $X^{2} h(0)>0$ and $Y^{2} h(0)>0$ (parabolic positive) or $X^{2} h(0)<0$ and $Y^{2} h(0)<0$ (parabolic negative), denoted by $\Omega_{4}^{p+}$ and $\Omega_{4}^{p-}$, resp.. We put $\Omega_{4}^{p}=\Omega_{4}^{p+} \cup \Omega_{4}^{p-}$;

(b) Hyperbolic case: $X^{2} h(0)>0$ and $Y^{2} h(0)<0$. We denote this set by $\Omega_{4}^{h}$;

(c) Elliptic case: $X^{2} h(0)<0$ and $Y^{2} h(0)>0$. We denote this set by $\Omega_{4}^{e}$.

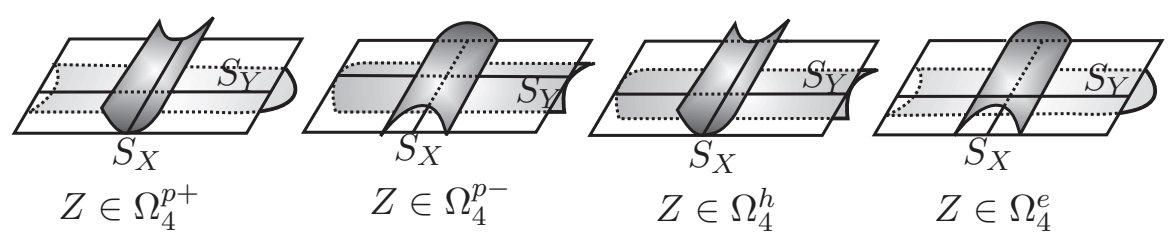

Figure 1. The kinds of two-fold singularities.

When a two-fold singularity is elliptic it is also called T-Singularity (or Teixeira-singularity) in the literature due to the pioneering work [15] .

The following construction is given in [16]. Let $Z=(X, Y) \in \Omega_{4}^{e}$. Applying the Implicit Function Theorem, for each $p \in(\Sigma, 0)$ there exists a smallest time $t(p)>0$ such that the orbit-solution $t \mapsto \phi_{X}(t, p)$ of $X$ through $p$ meets 
$\Sigma$ at a point $\widetilde{p}=\phi_{X}(t(p), p)$. We define the positive half-return map associated to $X$ by $\gamma_{X}:\left(\mathbb{R}^{2}, 0\right) \rightarrow\left(\mathbb{R}^{2}, 0\right)$ where $\gamma_{X}(p)=\widetilde{p}$. This map is a $C^{r}$-diffeomorphism and satisfies: $\gamma_{X}^{2}=I d$. Analogously, we define the positive half-return map associated to $Y$ by $\gamma_{Y}:\left(\mathbb{R}^{2}, 0\right) \rightarrow\left(\mathbb{R}^{2}, 0\right)$. The positive first return map associated to $Z=(X, Y)$ is given by $\varphi_{Z}:(\Sigma, 0) \rightarrow(\Sigma, 0)$, defined by the composition $\varphi_{Z}=\gamma_{Y} \circ \gamma_{X}$. Analogously we define the $n e$ gative half-return and the negative first return maps. Observe that we can change the order of $\gamma_{Y}$ and $\gamma_{X}$ and consequently we get another expression of $\varphi_{Z}$. However both expressions produce the same results and we fix the first one.

Let us define the sign function by $\operatorname{sgn}(0)=0, \operatorname{sgn}(x)=1$ if $x>0$, $\operatorname{sgn}(x)=-1$ if $x<0$.

\section{MAin RESUltS}

Given a PSVF $Z$, in our approach several ingredients and tools are used, such as: the contact between a vector field and the boundary of a manifold, sliding vector field, first return map associated to $Z$ and the interaction between these ingredients. The main goal of this paper is to exhibit explicitly the homeomorphisms that relate a locally structurally stable PSVF in $\Omega^{S}=\Omega_{0} \backslash \Omega_{4}^{e}$ with its respectively normal form. We exclude the set $\Omega_{4}^{e}$ because its behavior can be chaotic, see [7]. As consequence we obtain the main results of the paper:

Theorem A. If $Z \in \Omega^{S}$ then $Z$ is locally $\Sigma$-structurally stable.

The next result treats about the normal forms for the case where $Z \in$ $\Omega_{1} \cup \Omega_{2} \cup \Omega_{3}$.

Theorem B. If $Z \in \Omega_{\widetilde{1}} \cup \Omega_{2} \cup \Omega_{3}$ then $Z$ is $\Sigma$-equivalent to one of the following normal forms $\widetilde{Z}=(\widetilde{X}, \widetilde{Y})$, with:

(1) $\tilde{X}=\left(0,0, \varepsilon_{1}\right), \tilde{Y}=\left(0,0, \varepsilon_{1}\right)$, since $Z \in \Omega_{1}, \Sigma \equiv \Sigma^{c}$, where $\varepsilon_{1}=$ $\operatorname{sgn}(X h(0))$

(2) $\tilde{X}=\left(0,1, \varepsilon_{1}\right), \widetilde{Y}=\left(0,1,-\varepsilon_{1}\right)$, since $Z \in \Omega_{1}, \Sigma \equiv \Sigma^{s, e}$ and 0 is a $\Sigma$-regular point of $Z$, where $\varepsilon_{1}=\operatorname{sgn}(X h(0))$;

(3) $\widetilde{X}=\left(a x, b y, \varepsilon_{1}\right), \widetilde{Y}=\left(0,0,-\varepsilon_{1}\right)$, since $Z \in \Omega_{1}, \Sigma \equiv \Sigma^{s}$ (resp., $\left.\Sigma \equiv \Sigma^{e}\right)$ and 0 is a hyperbolic equilibrium of $Z^{\Sigma}$ (resp., $(-Z)^{\Sigma}$ ) such that the distinct eigenvalues $a=\lambda_{1}$ and $b=\lambda_{2}$ of $D Z^{\Sigma}(0)$ (resp., $\left.D(-Z)^{\Sigma}(0)\right)$ are real and nonzero, where $\varepsilon_{1}=\operatorname{sgn}(X h(0))$;

(4) $\widetilde{X}=\left(a x, b x, \varepsilon_{1}\right), \widetilde{Y}=\left(-b x, a y,-\varepsilon_{1}\right)$, since $Z \in \Omega_{1}, \Sigma \equiv \Sigma^{s}$ (resp., $\Sigma \equiv \Sigma^{e}$ ) and 0 is a hyperbolic focus of $Z^{\Sigma}$ (resp., $\left.(-Z)^{\Sigma}\right)$, where $a+i b=\lambda_{1}, a-i b=\lambda_{2}$ are the nonzero complex conjugate eigenvalues of $D Z^{\Sigma}(0)$ (resp., $\left.D(-Z)^{\Sigma}(0)\right)$, where $\varepsilon_{1}=\operatorname{sgn}(X h(0))$; 
(5) $\tilde{X}=(a, 0, x), \tilde{Y}=(1,0, b)$, since $Z \in \Omega_{2}$ where $a=\operatorname{sgn}\left(X^{2} h(0)\right)$ and $b=\operatorname{sgn}(Y h(0))$;

(6) $\widetilde{X}=\left(a, 0, b\left(y+x^{2}\right)\right), \widetilde{Y}=(1,0, c)$, since $Z \in \Omega_{3}$ where $a=\operatorname{sgn}\left(X^{1}(0)\right)$, $\operatorname{sgn}\left(X^{3} h(0)\right)$ and $c=\operatorname{sgn}(Y h(0))$.

In the following result we consider $Z \in \Omega_{4} \backslash \Omega_{4}^{e}$. Denote $E_{ \pm}$the eigenspaces generated by the eigenvectors of $D Z^{\Sigma}(0)$. Consider $p_{1}, p_{2}$ the fold points of $Z^{\Sigma}$. For more details, see Section 4.4 .

Theorem C. If $Z \in \Omega_{4} \backslash \Omega_{4}^{e}$ then $Z$ is $\Sigma$-equivalent to one of the following normal forms $\widetilde{Z}=(\widetilde{X}, \widetilde{Y})$, with:

(1) $\tilde{X}=(1,-2, x), \widetilde{Y}=(-1,1, y)$, since $Z \in \Omega_{4}^{p+}, Z^{\Sigma}$ presents a hyperbolic saddle singularity at the origin, $E_{ \pm} \subset \Sigma^{s} \cup \Sigma^{e}$ and $p_{1}, p_{2} \in \Sigma^{s}$;

(2) $\tilde{X}=(-1,2, x), \widetilde{Y}=(1,-1, y)$, since $Z \in \Omega_{4}^{p-}, Z^{\Sigma}$ presents a hyperbolic saddle singularity at the origin, $E_{ \pm} \subset \Sigma^{s} \cup \Sigma^{e}$ and $p_{1}, p_{2} \in \Sigma^{s}$;

(3) $\tilde{X}=(-1,-2, x), \widetilde{Y}=(-1,-1, y)$, since $Z \in \Omega_{4}^{p-}, Z^{\Sigma}$ presents a hyperbolic saddle singularity at the origin and $E_{ \pm} \subset \Sigma^{c}$;

(4) $\widetilde{X}=(1,2, x), \widetilde{Y}=(1,1, y)$, since $Z \in \Omega_{4}^{p+}, Z^{\Sigma}$ presents a hyperbolic saddle singularity at the origin and $E_{ \pm} \subset \Sigma^{c}$;

(5) $\widetilde{X}=(1,1 / 2, x), \widetilde{Y}=(-1,-1, y)$, since $Z \in \Omega_{4}^{h}, Z^{\Sigma}$ presents a hyperbolic saddle singularity at the origin, $E_{+} \subset \Sigma^{c}, E_{-} \subset \Sigma^{s} \cup \Sigma^{e}$ and $p_{1}, p_{2} \in \Sigma^{s}$

(6) $\widetilde{X}=(1,-1 / 2, x), \widetilde{Y}=(-1,-1, y)$, since $Z \in \Omega_{4}^{h}, Z^{\Sigma}$ presents a hyperbolic saddle singularity at the origin, $E_{+} \subset \Sigma^{c}, E_{-} \subset \Sigma^{s} \cup$ $\Sigma^{e}, p_{1} \notin \Sigma^{s}$ and $p_{2} \in \Sigma^{s}$;

(7) $\widetilde{X}=(1,1 / 2, x), \widetilde{Y}=(1,-1, y)$, since $Z \in \Omega_{4}^{h}, Z^{\Sigma}$ presents a hyperbolic saddle singularity at the origin, $E_{+} \subset \Sigma^{c}, E_{-} \subset \Sigma^{s} \cup \Sigma^{e}, p_{1} \in \Sigma^{s}$ and $p_{2} \notin \Sigma^{s}$;

(8) $\widetilde{X}=(1,-1 / 2, x), \widetilde{Y}=(1,-1, y)$, since $Z \in \Omega_{4}^{h}, Z^{\Sigma}$ presents a hyperbolic saddle singularity at the origin, $E_{+} \subset \Sigma^{c}, E_{-} \subset \Sigma^{s} \cup \Sigma^{e}, p_{1}, p_{2} \notin$ $\Sigma^{s}$

(9) $\widetilde{X}=(1,-2, x), \widetilde{Y}=(-1,1, y)$, since $Z \in \Omega_{4}^{p+}, Z^{\Sigma}$ presents a hyperbolic non-degenerated attractor node equilibrium at the origin and $E_{ \pm} \subset \Sigma^{s} \cup \Sigma^{e}$

(10) $\widetilde{X}=(1,-1, x), \widetilde{Y}=(-2,1, y)$, since $Z \in \Omega_{4}^{p+}, Z^{\Sigma}$ presents a hyperbolic non-degenerated repeller node equilibrium at the origin and $E_{ \pm} \subset \Sigma^{s} \cup \Sigma^{e}$

(11) $\widetilde{X}=(1,1, x), \widetilde{Y}=(2,1, y)$, since $Z \in \Omega_{4}^{p+}, Z^{\Sigma}$ presents a hyperbolic non-degenerated attractor node equilibrium at the origin and $E_{ \pm} \subset$ $\Sigma^{c}$

(12) $\widetilde{X}=(1,2, x), \widetilde{Y}=(1,1, y)$, since $Z \in \Omega_{4}^{p+}, Z^{\Sigma}$ presents a hyperbolic non-degenerated repeller node equilibrium at the origin and $E_{ \pm} \subset \Sigma^{c}$; 
(13) $\tilde{X}=(-1,1, x), \widetilde{Y}=(2,-1, y)$, since $Z \in \Omega_{4}^{p-}, Z^{\Sigma}$ presents a hyperbolic non-degenerated attractor node equilibrium at the origin and $E_{-} \subset \Sigma^{c}, E_{+} \subset \Sigma^{s} \cup \Sigma^{e}$

(14) $\widetilde{X}=(-1,2, x), \widetilde{Y}=(1,-1, y)$, since $Z \in \Omega_{4}^{p-}, Z^{\Sigma}$ presents a hyperbolic non-degenerated repeller node equilibrium at the origin and $E_{-} \subset \Sigma^{c}, E_{+} \subset \Sigma^{s} \cup \Sigma^{e}$

(15) $\widetilde{X}=(1,-2, x), \widetilde{Y}=(1,-1, y)$, since $Z \in \Omega_{4}^{h}, Z^{\Sigma}$ presents a hyperbolic non-degenerated attractor node equilibrium at the origin and $E_{-} \subset \Sigma^{c}, E_{+} \subset \Sigma^{s} \cup \Sigma^{e}$;

(16) $\widetilde{X}=(1,2, x), \widetilde{Y}=(-1,-1, y)$, since $Z \in \Omega_{4}^{h}, Z^{\Sigma}$ presents a hyperbolic non-degenerated repeller node equilibrium at the origin and $E_{-} \subset \Sigma^{c}, E_{+} \subset \Sigma^{s} \cup \Sigma^{e}$

(17) $\widetilde{X}=(1,-2, x), \widetilde{Y}=(-1,1, y)$, since $Z \in \Omega_{4}^{p+}, Z^{\Sigma}$ presents a hyperbolic non-degenerated attractor focus equilibrium at the origin;

(18) $\widetilde{X}=(1,-1, x), \widetilde{Y}=(-2,1, y)$, since $Z \in \Omega_{4}^{p+}, Z^{\Sigma}$ presents a hyperbolic non-degenerated repeller focus equilibrium at the origin;

(19) $\tilde{X}=(-1,1, x), \widetilde{Y}=(2,-1, y)$, since $Z \in \Omega_{4}^{p-}, Z^{\Sigma}$ presents a hyperbolic non-degenerated attractor focus equilibrium at the origin;

(20) $\widetilde{X}=(-1,2, x), \widetilde{Y}=(1,-1, y)$, since $Z \in \Omega_{4}^{p-}, Z^{\Sigma}$ presents a hyperbolic non-degenerated repeller focus equilibrium at the origin;

The Theorem A is obtained as consequence of Theorems B and C. In fact, consider two PSVF $Z$ and $\bar{Z}$ presenting the same behavior near the origin, for example, the origin is a fold-regular point for both. By Item 5 of Theorem B, we get that $Z$ and $\bar{Z}$ are $\Sigma$-equivalent to the normal form presented. Therefore, by a composition of $\Sigma$-equivalences we conclude that $Z$ and $\bar{Z}$ are $\Sigma$-equivalent. In the same way, we obtain the structural stability for the other cases presented in Theorem A.

Theorem B follows by Propositions 1, 2, 3 and Theorem C follows by Propositions 4, 5, 6 below.

\section{Structural Stability}

Following the same approach of $[1,2,5,9]$, in this section we construct explicitly the homeomorphism that conjugates a PSVF presenting some intrinsic conditions and the respectively normal form and consequently we prove the Theorems A, B and C.

The next propositions provide a proof of Theorems B and C. In what follows, we consider $U$ a neighborhood of the origin.

\subsection{Regular-regular Case.}

Proposition 1. Items (1), (2), (3) and (4) of Theorem B are true.

Proof. • In Item (1) we construct the homeomorphism by the composition of the flows $\phi_{X}, \phi_{Y}, \phi_{\tilde{X}}$ and $\phi_{\tilde{Y}}$. Consider $p \in \Sigma_{+}$, by the Implicit Function 
Theorem exists $t(p) \in \mathbb{R}$ such that $\phi_{X}(t(p), p) \in \Sigma$. Let

$$
\xi(p)= \begin{cases}\phi_{\widetilde{X}}\left(-t(p), \phi_{X}(t(p), p)\right), & \text { if } p \in \Sigma_{+} \\ p, & \text { if } p \in \Sigma ; \\ \phi_{\widetilde{Y}}\left(-t(p), \phi_{Y}(t(p), p)\right), & \text { if } p \in \Sigma_{-} .\end{cases}
$$

Observe that $\xi$ is of class $C^{0}$ and satisfies $\phi_{\widetilde{Z}}(t, \xi(p))=\xi\left(\phi_{Z}(t, p)\right)$.

- In Item (2), since the origin is a regular point of $\widetilde{Z}^{\Sigma}$, by the Flow Box Theorem there exists a homeomorphism $\bar{\xi}$ that locally conjugates the sliding vector fields $Z^{\Sigma}$ and $\widetilde{Z}^{\Sigma}$ on $\Sigma$. For points in $U \backslash \Sigma$ we extend the homeomorphism like in the previous case. The required homeomorphism is expressed by

$$
\xi(p)= \begin{cases}\phi_{\widetilde{X}}\left(-t(p), \bar{\xi}\left(\phi_{X}(t(p), p)\right)\right), & \text { if } p \in \Sigma_{+} \\ \bar{\xi}(p), & \text { if } p \in \Sigma \\ \phi_{\widetilde{Y}}\left(-t(p), \bar{\xi}\left(\phi_{Y}(t(p), p)\right)\right), & \text { if } p \in \Sigma_{-}\end{cases}
$$

- In Items (3) and (4), the origin is a hyperbolic critical point of $Z^{\Sigma}$ and of $\widetilde{Z}^{\Sigma}$. By the Hartman-Grobman Theorem there exists a homeomorphism $\bar{\xi}$ defined in a neighborhood $U \cap \Sigma$ that conjugates $Z^{\Sigma}$ and $\widetilde{Z}^{\Sigma}$. For points in $U \backslash \Sigma$ we can extend the homeomorphism like in the previous cases.

4.2. Fold-regular Case. We remind that $S_{X}$ is given implicitly by $\xi_{X}$ $\left(\xi_{X}^{-1}(0)=S_{X}\right)$, where $\xi_{X}(x, y)=x$.

Lemma 1. If $Z \in \Omega_{2}$ then $Z^{\Sigma}$ is transversal to $S_{X}$ at the origin.

Proof. By (2) we get the expression of $Z^{\Sigma}(x, y)$ and the contact between $Z^{\Sigma}$ and the boundary of $\Sigma^{s}$, at the origin, is given by $\left\langle Z^{\Sigma}(0), \nabla \xi_{X}(0)\right\rangle=$ $X^{1}(0) \neq 0$. Therefore, we conclude that $Z^{\Sigma}$ is transversal to $S_{X}$ at the origin.

Proposition 2. Item (5) of Theorem B is true.

Proof. Assume $Y h(0)>0$ (the case $Y h(0)<0$ is analogous). We have to analyze two cases: $(a) X^{2} h(0)<0$ and $(b) X^{2} h(0)>0$, see Figure 2 .

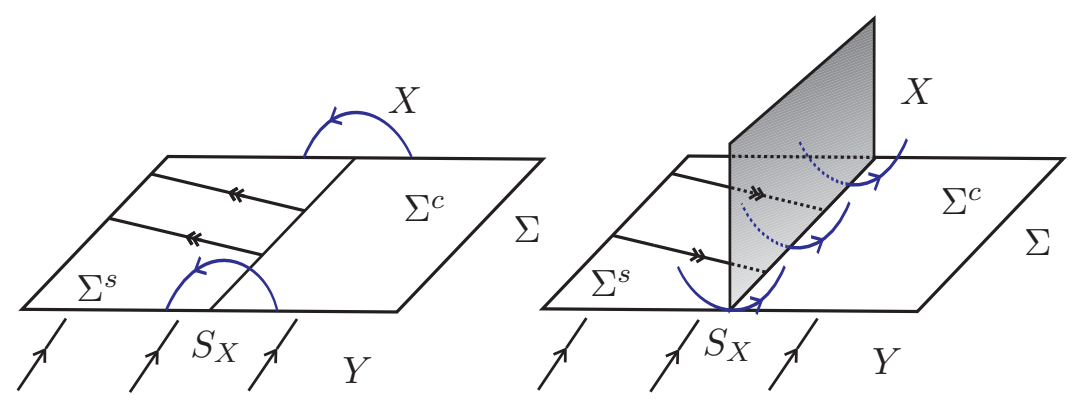

Case (a)

Case (b)

Figure 2. Positive and negative fold-regular cases. 
Case $(a)$ : let $S_{X} \cap U$, resp. $S_{\widetilde{X}} \cap U$ be the fold curves of $X$, resp. $\widetilde{X}$. Consider the homeomorphism $\xi: U \rightarrow U$ such that $\xi(0)=0$. By the arclength parametrization, we identify $S_{X}$ and $S_{\widetilde{X}}$. So, $\xi\left(S_{X}\right)=S_{\widetilde{X}}$. Given $p \in \Sigma^{s}$, by Lemma 1 , there exists $t_{1}(p) \in \mathbb{R}$ with $p_{1}=\phi_{Z^{\Sigma}}\left(t_{1}(p), p\right) \in S_{X}$. In this case we define $\xi(p)=\phi_{\widetilde{Z}_{\Sigma}}\left(-t_{1}(p), \xi\left(p_{1}\right)\right)$ where $\xi\left(p_{1}\right) \in S_{\widetilde{X}}$. For $p \in \Sigma^{c}$ there exists $t_{2}(p)>0$ such that $p_{2}=\phi_{X}\left(t_{2}(p), p\right) \in \Sigma^{s}$. In this case we define $\xi(p)=\phi_{\widetilde{X}}\left(-\widetilde{t}_{2}(p), \xi\left(p_{2}\right)\right)$ where $\xi\left(p_{2}\right) \in \Sigma^{c}$ and $\widetilde{t}_{2}(p)>0$ is the smallest time such that $\phi_{\widetilde{X}}\left(-\widetilde{t}_{2}(p), \xi\left(p_{2}\right)\right) \in \Sigma^{c}$. So, $\xi(\Sigma)=\Sigma$. Moreover, by arc-length parametrization $\xi\left(\Sigma_{+}\right)=\Sigma_{+}$.

If $p \in \Sigma_{-}$, by Implicit Function Theorem, there exists $t_{3}(p)>0$ with $p_{3}=\phi_{Y}\left(t_{3}(p), p\right) \in \Sigma$. In this case we define $\xi(p)=\phi_{\widetilde{X}}\left(-t_{3}(p), \xi\left(p_{3}\right)\right)$ where $\xi\left(p_{3}\right) \in \Sigma$. This concludes the proof of this case.

Case $(b)$ : the curves $S_{X}$ and $S_{\widetilde{X}}$ are composed by positive fold points. Define the homeomorphism $\xi: U \rightarrow U$ at $\bar{\Sigma}^{s}$ as in Case $(a)$. For $p \in \Sigma^{c}$, consider the trajectories of $X$ in $\Sigma_{-}$. So, there exists $t_{4}(p)<0$ such that $p_{4}=\phi_{X}\left(t_{4}(p), p\right) \in \Sigma^{s}$. In this case we define $\xi(p)=\phi_{\widetilde{X}}\left(-\widetilde{t}_{4}(p), \xi\left(p_{4}\right)\right)$ where $\xi\left(p_{4}\right) \in \Sigma^{s}$ and $-\widetilde{t}_{4}(p)>0$ is the smallest time such that $\phi_{\widetilde{X}}\left(-\widetilde{t}_{4}(p)\right.$, $\left.\xi\left(p_{4}\right)\right) \in \Sigma^{c}$. So, $\xi(\Sigma)=\Sigma$. Consider

$$
S_{X}^{\rho}=\left\{p \in \Sigma_{+} ; X h(p)=0 \text { and } d\left(p, S_{X}\right)=\rho\right\},
$$

where $d\left(p, S_{X}\right)$ is the euclidian distance between $p$ and $S_{X}$. Consider $p_{5}=$ $\left(x_{5}, y_{5}, z_{5}\right) \in S_{X}^{\rho}$ and $p_{5}^{*}=\left(x_{5}^{*}, y_{5}^{*}, 0\right) \in S_{X}$ such that $x_{5}=x_{5}^{*}$ (if it is necessary, we take a small neighborhood $\left.U_{1} \subset U\right)$. Define $\xi\left(p_{5}\right)=$ $\left(\xi_{1}\left(p_{5}^{*}\right), \xi_{2}\left(p_{5}^{*}\right), \rho\right)$, where $\xi\left(p_{5}^{*}\right)=\left(\xi_{1}\left(p_{5}^{*}\right), \xi_{2}\left(p_{5}^{*}\right), 0\right) \in S_{\widetilde{X}}$. In this way, $\xi\left(S_{X}^{\rho}\right)=S_{\widetilde{X}}^{\rho}=\{(0, y, \rho) ; y \in \mathbb{R}\} \cap U$. If $p_{6} \in\left(\left[\Sigma_{-} \cup \Sigma_{+}\right] \backslash\{X h=0\}\right)$, by Implicit Function Theorem, there exists $t_{6}\left(p_{6}\right) \in \mathbb{R}$ with either $p_{7}=$ $\phi_{Y}\left(t_{6}\left(p_{6}\right), p_{6}\right) \in \Sigma$ or $p_{7}=\phi_{X}\left(t_{6}\left(p_{6}\right), p_{6}\right) \in\{X h=0\}$. In this case we define either $\xi\left(p_{6}\right)=\phi_{\widetilde{Y}}\left(-t_{6}\left(p_{6}\right), \xi\left(p_{7}\right)\right)$ where $\xi\left(p_{7}\right) \in \Sigma$ or $\xi(p)=$ $\phi_{\widetilde{X}}\left(-t_{6}\left(p_{6}\right), \xi\left(p_{7}\right)\right)$ where $\xi\left(p_{7}\right) \in\{\widetilde{X} h=0\}$.

Therefore, we define the required homeomorphism $\xi: U \rightarrow U$ that provides the equivalence between $Z$ and $\widetilde{Z}$.

\subsection{Cusp-regular Case.}

Proposition 3. Item (6) of Theorem B is true.

Proof. Given $Z \in \Omega_{3}$, as we done in the proof of Lemma 1 we get that $Z^{\Sigma}$ is regular in a neighborhood of the origin. We suppose that $Y h(0)>0$ (the case $Y h(0)<0$ is analogous). The analysis depends on the sign of $X^{3} h(0)$ and $X_{1}(0)$. There are four cases to consider: $(a) X^{3} h(0)<0, X_{1}(0)<0$, (b) $X^{3} h(0)<0, X_{1}(0)>0,(c) X^{3} h(0)>0, X_{1}(0)<0$ and $(d) X^{3} h(0)>$ $0, X_{1}(0)>0$. In Cases $(a)$ and $(b)$ the dynamic of $Z$ is illustrated in Figure 3 .

Consider the Case $(a)$ and take an homeomorphism $\xi: U \rightarrow U$ such that $\xi(0)=0$. Observe that each one of the fold curves $S_{X}$ and $S_{\widetilde{X}}$ are 


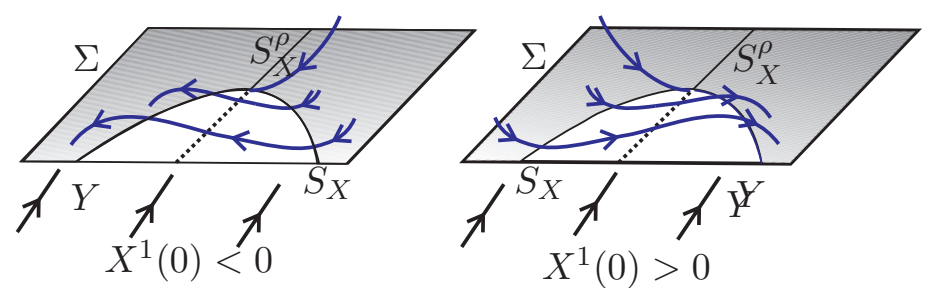

Figure 3. Cusp-regular case when $X^{3} h(0)<0$.

decomposed in two branches where the fold points are either positive or negative. We identify, by arc-length parametrization the branch of $S_{X}$ where the fold points are positive (resp., negative) and the branch of $S_{\widetilde{X}}$ where the fold points are positive (resp., negative). So, $\xi\left(S_{X}\right)=S_{\widetilde{X}}$.

Consider $\vec{n} \subset \Sigma$ a normal vector to $S_{X}$ at the cusp point, such that $\langle\vec{n}, \vec{n}\rangle=1$. We define, see Figure 3 , the set

$$
S_{X}^{\rho}=\left\{p \in \Sigma^{s} ; p=\rho \vec{n}\right\} .
$$

Given $p \in \Sigma^{s}$ there exists two possibilities:

(1) there exists $t_{1}(p) \in \mathbb{R}$ with $p_{1}=\phi_{Z^{\Sigma}}\left(t_{1}(p), p\right) \in S_{X}^{\rho}$ or

(2) there exists $t_{1}(p) \in \mathbb{R}$ with $p_{1}=\phi_{Z^{\Sigma}}\left(t_{1}(p), p\right) \in S_{X}$.

In the first case we define $\xi\left(p_{1}\right)=(0, \rho, 0) \in S_{\widetilde{X}}^{\rho}$. So, $\xi\left(S_{X}^{\rho}\right)=S_{\widetilde{X}}^{\rho}=$ $\{(0, y, 0) ; y=\rho\}$. In both cases we define: $\xi(p)=\phi_{\widetilde{Z}^{\Sigma}}\left(-\widetilde{t}_{1}(p), \xi\left(p_{1}\right)\right)$ and we get $\xi\left(\Sigma^{s}\right)=\Sigma^{s}$.

If $p_{2} \in \Sigma^{c}$ then there exists $t_{2}\left(p_{2}\right)>0$ the smallest time such that $p_{3}=$ $\phi_{X}\left(t_{2}\left(p_{2}\right), p_{2}\right) \in \Sigma^{s}$. Let $\widetilde{p}_{3}=\xi\left(p_{3}\right)$. So there exists, $-\widetilde{t}_{2}\left(\widetilde{p}_{3}\right)>0$ the smallest time such that $\widetilde{p}_{2}=\phi_{\widetilde{X}}\left(-\widetilde{t}_{2}\left(\widetilde{p}_{3}\right), \widetilde{p}_{3}\right) \in \Sigma^{c}$. We define $\xi\left(p_{2}\right)=\widetilde{p}_{2}$ and so $\xi(\Sigma)=\Sigma$.

Note that given $p_{4} \in U \backslash \Sigma$ then there exists $t_{4}\left(p_{4}\right)>0$ such that $\phi_{X}\left(t_{4}\left(p_{4}\right), p_{4}\right) \in \Sigma$ or $\phi_{Y}\left(t_{4}\left(p_{4}\right), p_{4}\right) \in \Sigma$. Therefore, we extend the homeomorphism for all neighborhood $U$ like in the previous propositions. The constructions for the other cases is similar and we omit.

4.4. Two-fold Case. In this subsection we consider the case where the origin is a fold point for both $X$ and $Y$. The structural stability for this case has been object of great interested in recent years (see $[6,7,10,11]$ among others). Teixeira exhibited in [15] a family of PSVF's $Z=(X, Y)$ presenting an elliptic two-fold singularity at the origin that is not structurally stable. So, since the purpose of this paper is to exhibit a subset of $\Omega_{4}$ composed by structurally stable PSVF's, we just consider $Z \in \Omega_{4}^{h} \cup \Omega_{4}^{p}$.

The curves $S_{X}$ and $S_{Y}$ split the switching manifold in 4 regions: $\Sigma^{s}, \Sigma^{e}, \Sigma^{c+}$ and $\Sigma^{c-}$. The position of these regions depends on the sign $\varepsilon_{1}$ and $\varepsilon_{2}$ of $X h$ and $Y h$, resp., see Figure 4. However, the topological structure in a neighborhood of the origin will not change, only the position of the regions on $\Sigma$. 


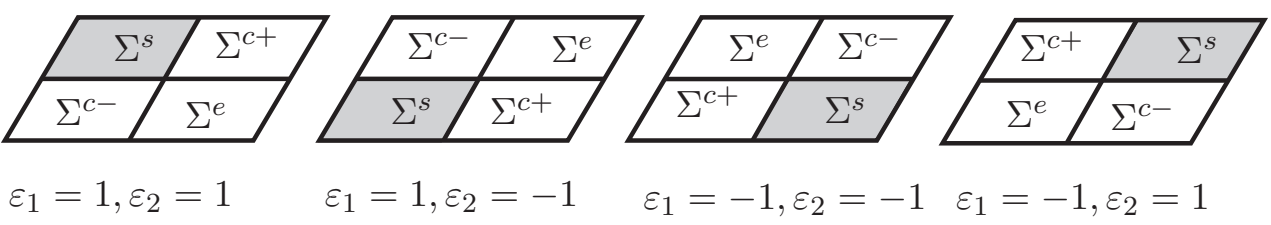

FiguRE 4. Regions on $\Sigma$, according the sign of $\varepsilon_{i}, i=1,2$.

Given $Z \in \Omega_{4}^{h} \cup \Omega_{4}^{p}$, we consider the following expression for $Z=(X, Y)$ :

$$
Z(x, y, z)=\left\{\begin{array}{l}
X(x, y, z)=\left(a, b, \varepsilon_{1} x\right) \\
Y(x, y, z)=\left(c, d, \varepsilon_{2} y\right)
\end{array}\right.
$$

where $\varepsilon_{i}= \pm 1, i=1,2, X^{2} h(0)=\varepsilon_{1} a, X Y h(0)=\varepsilon_{2} b, Y X h(0)=\varepsilon_{1} c$ and $Y^{2} h(0)=\varepsilon_{2} d$. The signs of $\varepsilon_{i}$ determinate the position of the regions on $\Sigma$, see Figure 4 , and the parameters $a, b, c$ and $d$ are determined according to the possible dynamics of $Z$. Here we consider only the case presented in Figure 4 as $\varepsilon_{1}=\varepsilon_{2}=1$. For the other cases (i.e., distinct positions of the regions) we get similar results.

A necessary condition for $Z \in \Omega^{r}$ be structurally stable in a neighborhood of the origin is that the associated sliding vector field $Z^{\Sigma}$ is structurally stable. If the origin is a hyperbolic singularity of $Z^{\Sigma}$, by Hartman-Grobman Theorem, we get the structurally stability of $Z^{\Sigma}$ in a neighborhood of the origin. By (2) and considering the expression (3) we obtain the expression for the sliding vector field $Z^{\Sigma}(x, y)=(-c x+a y,-d x+b y)$. The eigenvalues associated to $Z^{\Sigma}$ are

$$
\lambda_{ \pm}=\frac{(b-c) \pm \sqrt{\Delta}}{2}
$$

where $\Delta=(b+c)^{2}-4 a d$. The resp. eigenvectors are

$$
v_{ \pm}=\left(v_{ \pm}^{1}, v_{ \pm}^{2}\right)=\left(\frac{b+c \mp \sqrt{\Delta}}{2 d}, 1\right) .
$$

Let $E_{ \pm}$be the eigenspaces of $Z^{\Sigma}$ generated by the eigenvectors $v_{ \pm}$, respectively.

Note that some choices on these parameters leads to centers and degenerated nodes (when either $\Delta=0$ or some eigenvalue is null). Excluding these non-stable situations, we obtain the expressions of normal forms just in the cases where the origin is a hyperbolic singularity of type: saddle, focus and a non-degenerated node for $Z^{\Sigma}$.

4.4.1. Saddle singularity. Consider now the case where the origin is a hyperbolic saddle singularity of $Z^{\Sigma}$ where $Z \in \Omega_{4}^{p} \cup \Omega_{4}^{h}$. Note that the dynamic of $Z$ depends on the position of the eigenspaces $E_{ \pm}$of $Z^{\Sigma}$, i.e., $E_{ \pm} \subset \Sigma^{s} \cup \Sigma^{e}, E_{ \pm} \subset \Sigma^{c}$ or $E_{+} \subset \Sigma^{c}, E_{-} \subset \Sigma^{s} \cup \Sigma^{e}$. Considering (5) we get the position of the eigenspaces according to the sign of $d$ and $b+c$, see Figure 5. Given $U \subset \mathbb{R}^{3}$ a neighborhood of the origin, we consider a 


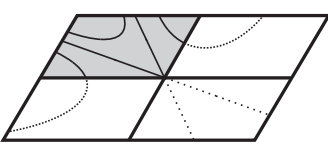

(a)

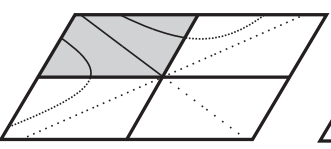

(b)

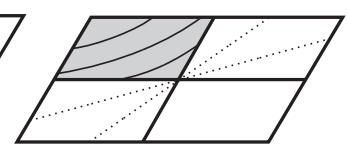

(c)

$E_{ \pm} \subset \Sigma^{s} \cup \Sigma^{e} \quad E_{+} \subset \Sigma^{c}, E_{-} \subset \Sigma^{s} \cup \Sigma^{e} \quad E_{ \pm} \subset \Sigma^{c}$

Figure 5. Possible dynamics of $Z^{\Sigma}$ when the origin is a hyperbolic saddle singularity.

box neighborhood of the origin $V_{\varepsilon}=I_{\varepsilon} \times I_{\varepsilon} \times I_{\varepsilon} \subset U$ where $I_{\varepsilon}=(-\varepsilon, \varepsilon)$ and $\varepsilon>0$ is small. An important conclusion concerning this case is the occurrence of tangencies between the trajectories of $Z^{\Sigma}$ and the boundary of $V_{\varepsilon} \cap \Sigma$, given in Figures 6 and 7, according to the position of eigenspaces.

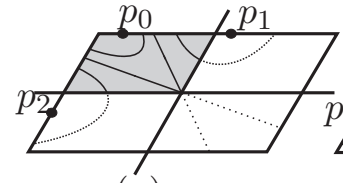

(a)

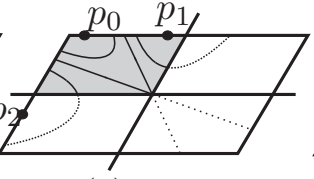

(b)

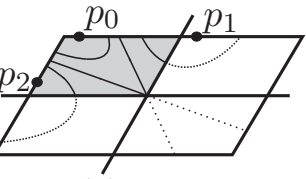

(c)

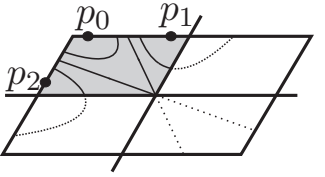

(d)

Figure 6. Possible tangencies of $Z^{\Sigma}$ for the case $E_{ \pm} \subset \Sigma^{s} \cup \Sigma^{e}$.
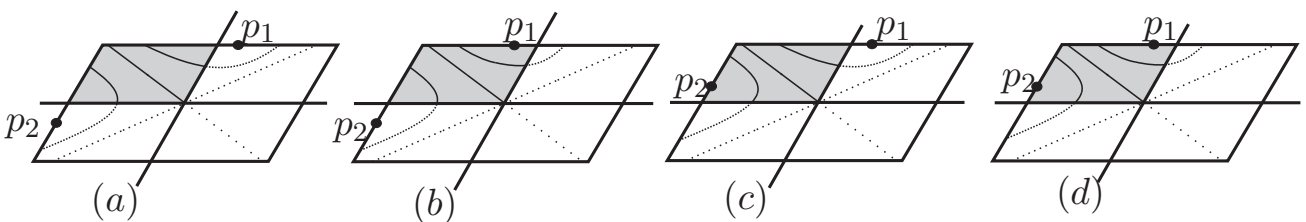

Figure 7. Possible tangencies of $Z^{\Sigma}$ for the case $E_{+} \subset$ $\Sigma^{c}, E_{-} \subset \Sigma^{s} \cup \Sigma^{e}$.

Notation: We denote the boundary of an arbitrary set $A$ by $\partial A$.

In order to study the contact between $Z^{\Sigma}$ and $\partial\left(V_{\varepsilon} \cap \Sigma\right)$, we define the functions: $\xi_{1}(x, y)=x+\varepsilon$ and $\xi_{2}(x, y)=y-\varepsilon$.

Lemma 2. Consider $Z \in \Omega^{r}$ such that $Z^{\Sigma}$ presents a hyperbolic saddle singularity at the origin. Then the trajectories of $Z^{\Sigma}$ are tangent to the straight lines $y=\varepsilon$ and $x=-\varepsilon$ at the points

$$
p_{1}=(b / d, 1) \varepsilon \text { and } p_{2}=(-1,-c / a) \varepsilon .
$$

Proof. The existence of tangencies of $Z^{\Sigma}$ with the straight line $y=\varepsilon$ (resp., $x=-\varepsilon)$ at $p_{1}$ (resp., $p_{2}$ ), is given by the conditions:

$$
Z^{\Sigma} \xi_{2}\left(p_{1}\right)=\left\langle Z^{\Sigma}\left(p_{1}\right), \xi_{2}\left(p_{1}\right)\right\rangle=0 \text { and }\left\langle Z^{\Sigma}\left(p_{1}\right), \nabla Z^{\Sigma} \xi_{2}\left(p_{1}\right)\right\rangle \neq 0
$$




$$
\text { (resp., } \left.Z^{\Sigma} \xi_{1}\left(p_{2}\right)=\left\langle Z^{\Sigma}\left(p_{2}\right), \xi_{1}\left(p_{2}\right)\right\rangle=0 \text { and }\left\langle Z^{\Sigma}\left(p_{2}\right), \nabla Z^{\Sigma} \xi_{1}\left(p_{2}\right)\right\rangle \neq 0\right) \text {. }
$$

Besides, these tangencies are invisible fold singularities of $Z^{\Sigma}$. Considering the expression of $Z$ given in (3) we get that the tangencies of $Z^{\Sigma}$ are: $p_{1}=$ $(b / d, 1) \varepsilon$ and $p_{2}=(-1,-c / a) \varepsilon$.

Remark 1. When $E_{ \pm} \subset \Sigma^{s} \cup \Sigma^{e}$, whose dynamic is presented in Figure 6, there exists a persistent tangency $p_{0}=\left(x_{0}, y_{0}\right) \in \mathcal{C}$ for $Z^{\Sigma}$, where $\mathcal{C} \subset \Sigma^{s}$ is the cone bounded by $E_{+} \cup E_{-} \cup \partial\left(V_{\varepsilon} \cap \Sigma\right)$. When $E_{+} \subset \Sigma^{c}$ and $E_{-} \subset \Sigma^{s} \cup \Sigma^{e}$, whose dynamics is presented in Figure 7 , the point $p_{0}$ does not exists.

In next lemma we prove that the possible dynamics presented in cases $(a),(b),(c)$ of Figure 6 do not occur.

Lemma 3. Consider $Z \in \Omega^{r}$ such that $Z^{\Sigma}$ presents a hyperbolic saddle singularity at the origin.

(1) If $E_{ \pm} \subset \Sigma^{s} \cup \Sigma^{e}$ then $p_{1}, p_{2} \in \Sigma^{s}$ and $Z \in \Omega_{4}^{p}$. Besides, if $d>0(d<$ $0)$ then $v_{+}^{1}<v_{-}^{1}\left(v_{+}^{1}>v_{-}^{1}\right)$, respectively.

(2) The configuration $E_{+} \subset \Sigma^{s} \cup \Sigma^{e}$ and $E_{-} \subset \Sigma^{c}$ does not occur.

Proof. - Item (1): If the origin is a hyperbolic saddle singularity of $Z^{\Sigma}$ and $E_{ \pm} \subset \Sigma^{s} \cup \Sigma^{e}$ then $0<a d<b c$.

Suppose that $d>0$ and $p_{1}, p_{2} \notin \Sigma^{s}$ then by (6) we conclude $b>0$ and $c>0$, which is a contradiction with $E_{ \pm} \subset \Sigma^{s} \cup \Sigma^{e}$. If $d<0$ and $p_{1}, p_{2} \notin \Sigma^{s}$ we conclude that $b<0$ and $c>0$. Which is a contraction with $0<a d<b c$. We get the same contradictions with the cases $p_{1} \in \Sigma^{s}, p_{2} \notin \Sigma^{s}$ and $p_{1} \notin \Sigma^{s}, p_{2} \in \Sigma^{s}$. Therefore, the only realizable case is when $p_{1}, p_{2} \in \Sigma^{s}$. The position of $v_{+}$and $v_{-}$follows by expression (5).

- Item (2): If the origin is a hyperbolic saddle singularity of $Z^{\Sigma}, E_{-} \subset \Sigma^{c}$ and $E_{+} \subset \Sigma^{s} \cup \Sigma^{e}$ then $d>0$ and $a<0$. Which is a contraction with $Z \in \Omega_{4}^{h} \cup \Omega_{4}^{p}$.

In the next lemma consider $p_{i}, i=0,1,2$ the fold singularities of $Z^{\Sigma}$, defined in (6) and Remark 1 , and $\widetilde{p}_{i}, i=0,1,2$, the resp. fold singularities of $\widetilde{Z}^{\Sigma}$.

Lemma 4. If there exists $\xi: \mathbb{R}^{3}, 0 \rightarrow \mathbb{R}^{3}, 0$ a $\Sigma$-equivalence between $Z$ and $\widetilde{Z}$ then $\xi\left(p_{i}\right)=\widetilde{p}_{i}, i=0,1,2$.

In other words, Lemma 4 tell us that the tangential singularities of $Z^{\Sigma}$ are preserved by $\Sigma$-equivalences.

Proof. In Figures 6 and 7 we get the possible dynamics of $Z^{\Sigma}$. However, in Lemma 3 we prove that the dynamics that really occur are only that presented in Figures 5- $(c), 6-(d)$ and in Figure 7 with $E_{-} \subset \Sigma^{s} \cup \Sigma^{e}$ and $E_{+} \subset \Sigma^{c}$. Therefore, we have to prove that the dynamics presented in these figures are not $\Sigma$-equivalent. We detail the proof for the dynamics given in Figure $7(a)$ and $(d)$. The proof for the other cases is similar and we omit. 
Consider $Z$ and $\widetilde{Z}$ presenting the dynamics of Figure $7(a)$ and $(d)$, respectively. Let $p_{-}=E_{-} \cap \partial\left[V_{\varepsilon} \cap \Sigma^{s}\right], p_{X}=(-\varepsilon, 0,0) \in S_{Y} \cap \partial V_{\varepsilon}$ and $p_{Y}=(0, \varepsilon, 0) \in S_{X} \cap \partial V_{\varepsilon}$ and the resp. points associated to the dynamic of $\widetilde{Z}$, see Figure 8. We define $S_{X}^{+}=S_{X} \cap\{(x, y, 0) ; y>0\}$ and $\widetilde{p}_{3} \in$ $\partial\left[V_{\varepsilon} \cap \Sigma^{s}\right]$ such that $\phi_{\widetilde{Z}^{\Sigma}}\left(\widetilde{p}_{3}, t^{*}\right)=p_{\widetilde{Y}}$. Note that there exists $t^{*}$ satisfying the last equality since $\widetilde{p}_{1}$ is an invisible fold singularity for $\widetilde{Z}^{\Sigma}$. We have that

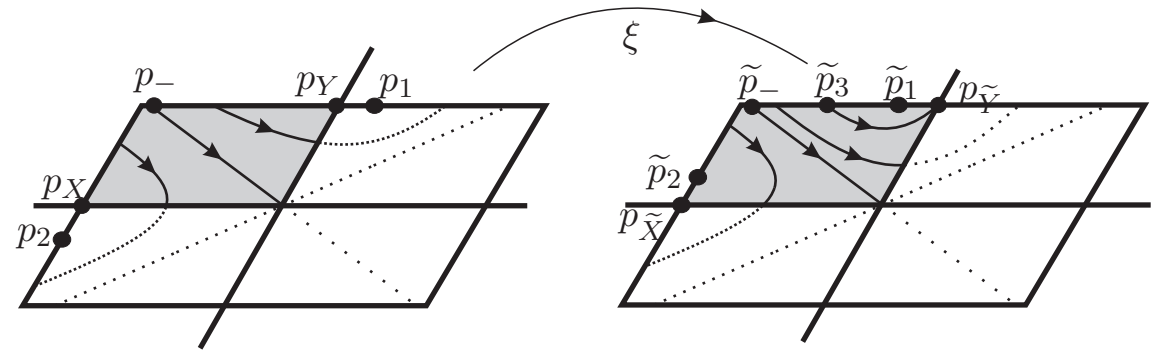

FiguRE 8 . The $\Sigma$-equivalence must preserve the fold points of $Z^{\Sigma}$.

$\phi_{Z^{\Sigma}}\left(S_{X}^{+}\right) \cap \partial\left[V_{\varepsilon} \cap \Sigma^{s}\right]=\left(p_{-}, p_{Y}\right)$ and $\phi_{\widetilde{Z}^{\Sigma}}\left(S_{\widetilde{X}}^{+}\right) \cap \partial\left[V_{\varepsilon} \cap \Sigma^{s}\right]=\left(\widetilde{p}_{-}, \widetilde{p}_{3}\right]$. So, $\phi_{\widetilde{Z}^{\Sigma}}\left(\xi\left(S_{X}^{+}\right)\right) \cap \partial\left[V_{\varepsilon} \cap \Sigma^{s}\right]=\phi_{\widetilde{Z}^{\Sigma}}\left(S_{\widetilde{X}}^{+}\right) \cap \partial\left[V_{\varepsilon} \cap \Sigma^{s}\right]=\left(\widetilde{p}_{-}, \widetilde{p}_{3}\right]$ and $\xi\left(\phi_{Z^{\Sigma}}\left(S_{X}^{+}\right) \cap\right.$ $\left.\partial\left[V_{\varepsilon} \cap \Sigma\right]\right)=\xi\left(\left(p_{-}, p_{Y}\right]\right)=\left(\widetilde{p}_{-}, p_{\widetilde{Y}}\right)$. However, $\left(\widetilde{p}_{-}, \widetilde{p}_{3}\right] \varsubsetneqq\left(\widetilde{p}_{-}, p_{\widetilde{Y}}\right)$. Which is a contradiction with the hypothesis that $\xi$ is an equivalence between $Z$ and $\widetilde{Z}$. Therefore, we conclude that $Z$ and $\widetilde{Z}$ are not $\Sigma$-equivalent.

Proposition 4. Items (1), ., (8) of Theorem $C$ are true.

Proof. We detail the construction of the $\Sigma$-equivalence for the cases presented in Items (1) and (2) of Theorem C. The construction for the other cases are similar and we omit.

- Item (1): We suppose that the origin is a hyperbolic saddle singularity for $Z^{\Sigma}$, the eigenspaces $E_{ \pm} \subset \Sigma^{s} \cup \Sigma^{e}$ and $p_{1}, p_{2} \in \Sigma^{s}$. By (6) we conclude that the origin is a parabolic two-fold singularity of $Z=(X, Y)$. Let $S_{X}, S_{Y}$, resp. $S_{\widetilde{X}}, S_{\widetilde{Y}}$ be the fold curves of $X, Y$, resp. $\widetilde{X}, \widetilde{Y}$. Consider the homeomorphism $\xi: V_{\varepsilon} \rightarrow V_{\varepsilon}$ such that $\xi(0)=0$. By the arc-length parametrization, we define $\xi\left(S_{X}\right)=S_{\widetilde{X}}$ and $\xi\left(S_{Y}\right)=S_{\widetilde{Y}}$. The eigenspaces $E_{ \pm}$and $\widetilde{E}_{ \pm}$, in $V_{\varepsilon}$, are invariant by $Z^{\Sigma}$ and $\widetilde{Z}^{\Sigma}$, resp., so we identify $\xi\left(E_{ \pm}\right)=\widetilde{E}_{ \pm}$.

Let $p_{ \pm}=E_{ \pm} \cap \partial\left[V_{\varepsilon} \cap \Sigma^{s}\right], p_{Y}=(-\varepsilon, 0,0) \in S_{Y} \cap \partial V_{\varepsilon}$ and $p_{X}=(0, \varepsilon, 0) \in$ $S_{X} \cap \partial V_{\varepsilon}$. Consider the segments $\left[p_{0}, p_{-}\right],\left[p_{Y}, p_{2}\right],\left[p_{1}, p_{X}\right] \subset \partial\left[V_{\varepsilon} \cap \Sigma\right]$. There are similar segments $\left[\widetilde{p}_{0}, \widetilde{p}_{-}\right],\left[p_{\widetilde{Y}}, \widetilde{p}_{2}\right],\left[\widetilde{p}_{1}, p_{\widetilde{X}}\right] \subset \partial\left[V_{\varepsilon} \cap \Sigma\right]$, where $\widetilde{p}_{0}$ is a persistent fold singularity of $\widetilde{Z}^{\Sigma}, \widetilde{p}_{1}=(-2,1,0) \varepsilon, \widetilde{p}_{2}=(-1,1,0) \varepsilon, \widetilde{p}_{-}=$ $((-3+\sqrt{5}) / 2,1,0) \varepsilon, p_{\widetilde{X}}=(0, \varepsilon, 0)$ and $p_{\widetilde{Y}}=(-\varepsilon, 0,0)$.

By arc length parametrization we extend $\xi$ for these segments: $\xi\left(\left[p_{0}, p_{-}\right]\right)$ $=\left[\widetilde{p}_{0}, \widetilde{p}_{-}\right], \xi\left(\left[p_{Y}, p_{2}\right]\right)=\left[p_{\tilde{Y}}, \widetilde{p}_{2}\right]$ and $\xi\left(\left[p_{1}, p_{X}\right]\right)=\left[\widetilde{p}_{1}, p_{\tilde{X}}\right]$. Using the flows of $Z^{\Sigma}$ and $\widetilde{Z}^{\Sigma}$ we can extend $\xi$ for all $\bar{\Sigma}^{s}$, as we done in Subsection 4.2. 
In order to extend the homeomorphism $\xi$ for $\Sigma^{c+}$, we consider a virtual dynamics of $Z^{\Sigma}$ in $\Sigma^{c+}$. In fact, in (1) we defined $Z^{\Sigma}$ only in $\Sigma^{s}$, however by this expression we can consider its dynamics in all neighborhood $\Sigma \cap V_{\varepsilon} \subset$ $\left(\mathbb{R}^{2}, 0\right)$. Of course, outside of $\Sigma^{s}$ this dynamics it is not realizable. Hence, we use the term virtual dynamics of $Z^{\Sigma}$.

The virtual dynamic of $Z^{\Sigma}$ in $\Sigma^{c+}$ is transient, i.e., given $p \in \Sigma^{c+}$ there exists $t(p) \in \mathbb{R}$ such that $\phi_{Z^{\Sigma}}(t(p), p) \in S_{X}$. Therefore, we extend $\xi$ for $\Sigma^{c+}$ by the virtual flows of $Z^{\Sigma}$ and $\widetilde{Z}^{\Sigma}$ in $\Sigma^{c+}$. Given $p \in \Sigma^{c-} \cup \Sigma^{e}$ there exists $t(p) \in \mathbb{R}$ such that $\phi_{Y}(t(p), p) \in \Sigma^{c+} \cup \Sigma^{s}$. So, we extend $\xi$ for all $\Sigma$ and for $\Sigma_{-}$by the flows of $Y$ and $\widetilde{Y}$.

In order to extend $\xi$ for all $V_{\varepsilon}$, it is enough define $\xi$ in $\Sigma_{+}$, repeating the same procedure done in Subsection 4.2. Therefore, we get the $\Sigma$-equivalence between $Z$ and $\widetilde{Z}=(\widetilde{X}, \widetilde{Y})$, where $\widetilde{X}(x, y, z)=(1,-2, x)$ and $\widetilde{Y}(x, y, z)=$ $(-1,1, y)$, with $(x, y, z) \in V_{\varepsilon}$.

- Item (2): We suppose that the origin is a parabolic two-fold singularity of $Z=(X, Y)$, a hyperbolic saddle singularity for $Z^{\Sigma}$ and the eigenspaces $E_{ \pm} \subset \Sigma^{c}$, see Figure 5. In this case, $Z^{\Sigma}$ has a transient behavior in $\Sigma^{s}$. We define $\xi(0)=0$ and $\xi\left(S_{Y}\right)=S_{\widetilde{Y}}$. Using the flows of $Z^{\Sigma}$ and $\widetilde{Z}^{\Sigma}$ (in $\Sigma^{s}$ ) we extend $\xi$ for all $\Sigma^{s}$. Analogously to the previous case we can extend $\xi$ for $V_{\varepsilon}$ considering the virtual dynamics of $Z^{\Sigma}$ and the dynamics of $X$ and $Y$.

4.4.2. Node singularity. Consider now the case where the origin is a hyperbolic node of $Z^{\Sigma}$ where $Z \in \Omega_{4}^{p} \cup \Omega_{4}^{h}$. The dynamic of $Z$ depends on the stability of the node and, as in Subsection 4.4.1, of the position of eigenspaces $E_{ \pm}$. In this way the dynamic of $Z^{\Sigma}$ is one of those illustrated in Figure 9. In next lemma we prove that the possible dynamics

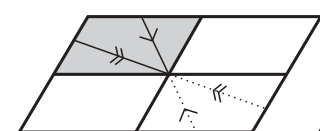

(1.a)

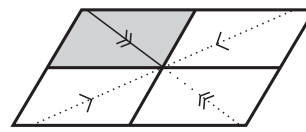

(2.a)

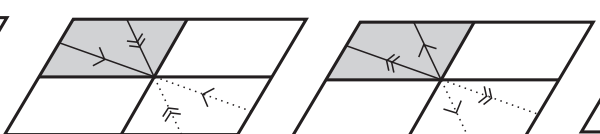

(1.b)

(1.c)

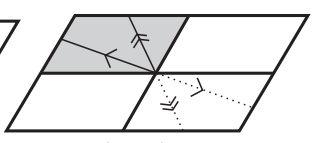

(1.d)

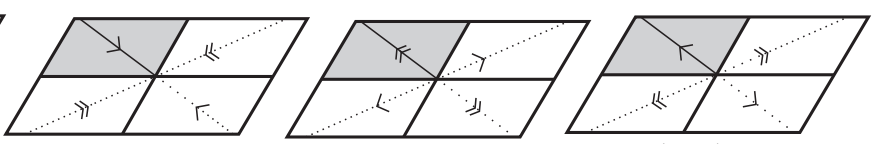

(2.b)

(2.d)

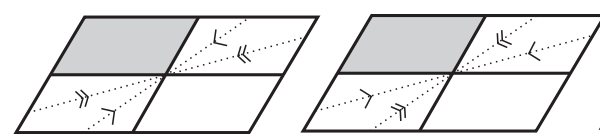

(3.b)

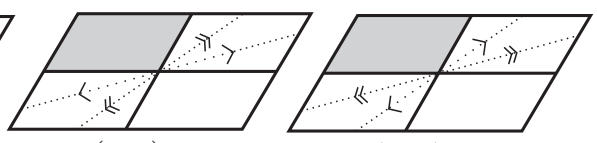

(3.c) (3.d)

Figure 9. Possible arrangements of the eigenspaces of $Z^{\Sigma}$ when the origin is a hyperbolic node. 
presented in cases $(1 . a),(1 . d),(2 . a),(2 . b),(2 . c),(2 . d),(3 . b)$ and $(3 . d)$ of Figure 9 do not occur when the origin is a parabolic positive two-fold singularity and cases $(1 . a),(1 . b),(1 . c),(1 . d),(2 . b),(2 . c),(3 . a),(3 . b),(3 . c)$ and (3.d) do not occur when the origin is a parabolic negative two-fold singularity.

We recall that $\Delta=(b+c)^{2}-4 a d$, defined in (4).

Lemma 5. Let the origin be a hyperbolic node of $Z^{\Sigma}$ where $Z \in \Omega_{4}^{p} \cup \Omega_{4}^{h}$, it holds:

(1) The eigenspace associated to the eigenvalue of biggest modulus is $E_{-}$, resp. $E_{+}$, when the node is attractor, resp. repeller.

(2) If the origin is a hyperbolic two-fold singularity then $E_{-} \subset \Sigma^{c}$ and $E_{+} \subset \Sigma^{s} \cup \Sigma^{e}$.

(3) If the origin is a parabolic positive two-fold singularity, either

(3.a) the eigenspaces $E_{ \pm} \subset \Sigma^{s} \cup \Sigma^{e}$ and they are placed counterclockwise in $\Sigma^{s} \cup \Sigma^{e}$; or

(3.b) $E_{ \pm} \subset \Sigma^{c}$ and they are placed counterclockwise in $\Sigma^{c}$.

(4) If the origin is a parabolic negative two-fold singularity $E_{+} \subset \Sigma^{c}$ and $E_{-} \subset \Sigma^{s} \cup \Sigma^{e}$.

Proof. • Item (1): Since the origin is a hyperbolic node, both eigenvalues $\lambda_{ \pm}$are real and have the same sign, i.e., $|b-c|>\sqrt{\Delta}>0$. In this way, if $b<c$, resp. $b>c$, then the node is attractor, resp. repeller, and the eigenspace associated to the eigenvalue of biggest modulus is $E_{-}$, resp. $E_{+}$.

- Item (2): We get, $\lambda_{+} \lambda_{-}>0$ and $a d>b c$. When $b>c$, both eigenvalues $\lambda_{ \pm}$are positive and the node is repeller. When $b<c$, both $\lambda_{ \pm}$are negative and the node is attractor. Besides, as the origin is a hyperbolic two-fold for $Z$, we get $X^{2} h(0)=a>0$ and $Y^{2} h(0)=d<0$. Therefore, $E_{+} \subset \Sigma^{s} \cup \Sigma^{e}$ and $E_{-} \subset \Sigma^{c}$. The local dynamics of $Z^{\Sigma}$ are presented in Figure 9 (2.b) and (2.c).

- Items (3) and (4): Considering the expression (5), we get $v_{+}^{1}<v_{-}^{1}$ when $d>0$ and $v_{+}^{1}>v_{-}^{1}$ when $d<0$. Moreover, $v_{+}^{1} v_{-}^{1}=\left((b+c)^{2}-\Delta\right) /\left(4 d^{2}\right)$. So, $(b+c)^{2}-\Delta>0$, resp. $<0$, when $v_{+}^{1}$ and $v_{-}^{1}$ have the same sign, resp. opposite sign. Taking, without loss of generality, $d=1$ in (5) we get that $(b+c)^{2}<\Delta=(b+c)^{2}-4 a$ does not holds because $a>0$. Analogously, taking $d=-1$ in (5) we get that $(b+c)^{2}>\Delta=(b+c)^{2}+4 a$ does not holds because $a<0$. Therefore, Item (3.a) holds when $v_{-}^{1}<0$ and, consequently, $2\left(v_{+}^{1}+v_{-}^{1}\right)=(b+c) / d<0$. Item $(3 . b)$ holds when $0<v_{+}^{1}$ and, consequently, $2\left(v_{+}^{1}+v_{-}^{1}\right)=(b+c) / d>0$. Item (4) holds when $v_{-}^{1}<0<v_{+}^{1}$.

Proposition 5. Items (9), .,,(16) of Theorem $C$ are true.

Proof. The construction of the $\Sigma$-equivalence in these cases are analogous to that ones in Proposition 4 considering the virtual dynamic of $Z^{\Sigma}$, the dynamic of $X$ and $Y$. 
4.4.3. Focus singularity. Consider now the case where the origin is a hyperbolic focus of $Z^{\Sigma}$ where $Z \in \Omega_{4}^{p} \cup \Omega_{4}^{h}$. Note that the dynamic of $Z$ depends on the orientation of $Z^{\Sigma}$ and on the sense that the trajectories of $Z^{\Sigma}$ encircle the origin, see Figure 10.
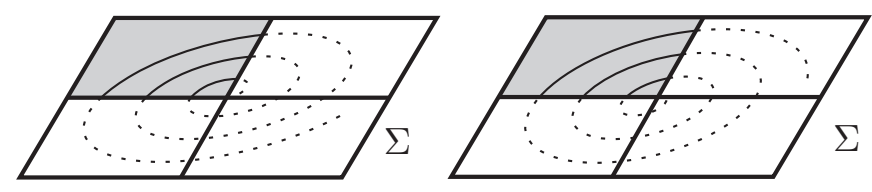

FIGURE 10. The trajectories of $Z^{\Sigma}$ can encircle the origin either clockwise or counterclockwise.

Proposition 6. Items $(17), \ldots,(20)$ of Theorem $C$ are true.

Proof. Since the imaginary part of the eigenvalues are non null and $\Delta<0$, we get that $(b+c)^{2}<4 a d$ and therefore, the origin is a parabolic two-fold singularity of $Z$. In this way, according to the sign of the real and the imaginary parts of the eigenvalues, we conclude that $(b-c)<0$ when 0 is an attractor focus, $(b-c)>0$ when 0 is a repeller focus, $a<0$ when the trajectories of $Z^{\Sigma}$ surround 0 in the clockwise direction and $a>0$ when the trajectories of $Z^{\Sigma}$ surround 0 in the counterclockwise direction.

We can define the $\Sigma$-equivalence between $Z$ and $\widetilde{Z}$ following the proof of Proposition 4 considering the virtual dynamic of $Z^{\Sigma}$, the dynamic of $X$ and $Y$.

Acknowledgments. First of all the authors would like to thank the professor Marco Antonio Teixeira for many helpful comments and suggestions in this paper. The first author was partially supported by FAPESP-BRAZIL grants 2012/00481-6 and 2014/02134-7 and the second is supported by FAPEG-Brazil Project 2012/10 267000803 and CNPq Project 478230/2013-3. This work is partially realized at UFG as a part of project numbers 35796 and 35797 .

\section{REFERENCES}

[1] Buzzi C.A., Carvalho T. de, Teixeira M.A., On 3-parameter families of piecewise smooth vector fields in the plane, SIAM Journal on Applied Dynamical Systems, v. 11, p. 1402-1424, 2012.

[2] Buzzi C.A., Carvalho T. de, Teixeira M.A., On three-parameter families of Filippov systems: The fold-saddle singularity, International Journal of Bifurcation and Chaos in Applied Sciences and Engineering, v. 22, p. 1250291, 2012.

[3] Buzzi C.A., Carvalho T. de, Teixeira M.A., Birth of limit cycles bifurcating from a nonsmooth center, Journal de Mathematiques Pures et Appliquees, volume 102, 36-47, 2014. 
[4] Carvalho T., Tonon D.J., Generic Bifurcations of Planar Filippov Systems via Geometric Singular Perturbations, Bull. Belg. Math. Soc. Simon Stevin 18, 861-881, 2011.

[5] Carvalho T., Tonon D.J., Normal Forms for Codimension One Planar Piecewise Smooth Vector Fields, Int. J. Bifurcation Chaos, vol. 24, 2014.

[6] Colombo A., Jeffrey M.R., The two-fold singularity of discontinuous vector fields, SIAM J. Appl. Dyn. Syst. vol. 8, Issue 2, pp. 624-640, 2009.

[7] Colombo A., Jeffrey M.R., Nondeterministic chaos, and the two-fold singularity in piecewise smooth flows, SIAM J. Appl. Dyn. Syst. vol. 10, pp. 423-451, 2011.

[8] Filippov A. F., Differential equations with discontinuous righthand sides, vol. 18 of Mathematics and its Applications (Soviet Series), Kluwer Academic Publishers Group, Dordrecht, 1988.

[9] Guardia M., Seara T.M., Teixeira, M.A., Generic bifurcations of low codimension of planar Filippov Systems, J. Diff. Eq., vol. 250, 1967-2023, 2011.

[10] Jacquemard A., Teixeira M.A. and Tonon D.J., Piecewise smooth reversible dynamical systems at a two-fold singularity, Int. Journal of Bifurcation and Chaos, vol. 22, n.8, 2012.

[11] Jacquemard A., Teixeira M.A. and Tonon D.J., Stability conditions in piecewise smooth dynamical systems at a two-fold singularity, Journal of Dynamical and Control Systems, vol. 19, No. 1, 47-67, 2013.

[12] Kuznetsov YU.A., Rinaldi S., Gragnani A., One-parameter bifurcations in planar Filippov Systems, Int. Journal of Bifurcation and Chaos, vol. 13, 2157-2188, 2003.

[13] Martins, R.M., Formal equivalence between normal forms of reversible and hamiltonian dynamical systems, Communications on Pure and Applied Analysis, vol. 13, 703-713, 2014.

[14] Sotomayor J., Teixeira M. A., Vector fields near the boundary of a 3manifold, Lect. Notes in Math., vol. 331, Springer Verlag, 169-195, 1988.

[15] Teixeira M. A., Stability conditions for discontinuous vector fields, J. Diff. Eq., vol. 88, 15-29, 1990.

[16] Teixeira M. A., Perturbation theory for non-smooth systems, Encyclopedia of Complexity and Systems Science, Springer, 2009.

[17] Teixeira M. A., Generic bifurcations of sliding vector fields, Journal of Mathematical Analysis and Applications, vol. 176, 436-457, 1993.

1 Departamento de Matemática, Faculdade de CiÊncias, Unesp, Av. Eng. Luiz Edmundo Carrijo Coube 14-01, CEP 17033-360, Bauru, SP, Brazil.

2 IME-UFG, CEP 74001-970, GoiÂnia, GoiÁs, BrazIL.

E-mail address: tcarvalho@fc.unesp.br

E-mail address: djtonon@ufg.br 